Article

\title{
Effect of Low Spin Excited States for Magnetic Anisotropy of Transition Metal Mononuclear Single Molecule Magnets
}

\author{
Leonel Llanos and Daniel Aravena * \\ Departamento de Química de los Materiales, Facultad de Química y Biología, Universidad de Santiago de \\ Chile (USACH), Casilla 40, Correo 33, Santiago 9170022, Chile; leonel.llanos@usach.cl \\ * Correspondence: daniel.aravena.p@usach.cl; Tel.: +56-227181179
}

Received: 14 December 2017; Accepted: 25 January 2018; Published: 27 January 2018

\begin{abstract}
Rational, fine tuning of magnetic anisotropy is critical to obtain new coordination compounds with enhanced single molecule magnet properties. For mononuclear transition metal complexes, the largest contribution to zero-field splitting is usually related to the excited states of the same spin as the ground level. Thus, the contribution of lower multiplicity roots tends to be overlooked due to its lower magnitude. In this article, we explore the role of lower multiplicity excited states in zero-field splitting parameters in model structures of $\mathrm{Fe}(\mathrm{II})$ and $\mathrm{Co}(\mathrm{II})$. Model aquo complexes with coordination numbers ranging from 2 to 6 were constructed. The magnetic anisotropy was calculated by state of the art ab initio methodologies, including spin-orbit coupling effects. For non-degenerate ground states, contributions to the zero-field splitting parameter $(D)$ from highest and lower multiplicity roots were of the same sign. In addition, their relative magnitude was in a relatively narrow range, irrespective of the coordination geometry. For degenerate ground states, the contribution from lower multiplicity roots was significantly smaller. Results are rationalized in terms of general expressions for $D$ and are expected to be reasonably transferable to real molecular systems.
\end{abstract}

Keywords: CASSCF calculations; magnetic anisotropy; single molecule magnets

\section{Introduction}

The discovery of mononuclear single molecule magnets [1] (also known as single ion magnets) led to new opportunities for the interplay of theoretical methods in this field. Concretely, complete active space self-consistent field (CASSCF) calculations permitted the direct calculation of magnetic anisotropy, which is the physical phenomenon responsible for the development of an energy barrier blocking magnetic relaxation. Nowadays, CASSCF calculations are widely applied for the rationalization of magnetic properties of mononuclear single molecule magnets based on transition metal ions and lanthanides [2-18]. Besides energies and wave functions, ab initio calculations can be analyzed in terms of Spin-Hamiltonian and ligand field parameters [19-21]. In this way, experimentally derived parameters as zero-field splitting $D$ and $E$, effective $g$-factors, and Racah and ligand field parameters can be directly compared with their calculated counterparts. In many cases, data from magnetic measurements is not enough to fit a consistent parameter set in a univocal way, which can be estimated by electronic structure methods [22-24].

One of the critical parameters to optimize in the quest for single molecule magnets operating at higher temperatures is the height of the demagnetization barrier. In the double well model for magnetic moment inversion [25], the demagnetization barrier $(U)$ is proportional to the zero-field splitting parameter $D$. Concretely, $U=D S^{2}$ and $U=D S^{2}-1 / 4$ for integer and half-integer systems, 
respectively. Although magnetic relaxation depends on several mechanisms [26-29], the fine tuning of magnetic anisotropy remains critical to improve the performance of single molecule magnets.

Concerning transition metal single molecule magnets, the relation between molecular structure and zero-field splitting has been explored [13,30-34]. These studies are useful to rationalize magnetic relaxation properties of new examples of single molecule magnets and to spot simple design rules to synthesize new systems with enhanced properties.

In most cases, magnetic anisotropy can be understood from the contributions of excited states of the same spin $(\mathrm{S} \rightarrow \mathrm{S})$ as the ground level, which are mixed by the spin-orbit coupling (SOC) operator. Understandably, excited states of other multiplicities $(S \rightarrow S \pm 1)$ are often not considered due to their lower contribution to $D$. An exception to the prevalence of $S \rightarrow S$ contributions are, of course, high-spin $\mathrm{Fe}(\mathrm{III})$ complexes, where magnetic anisotropy stems from sextet-quartet excitations. There are some examples of multireference ab initio studies on magnetic anisotropy of $\mathrm{d}^{5}$ systems [35-37] and one example of a mononuclear single molecule magnet featuring a high spin Fe(III) center [38]. Thus, it is interesting to explore the role of $S \rightarrow S-1$ contributions in the magnetic anisotropy of transition metal single molecule magnets, as they will be always present and could be beneficial or detrimental for magnetization relaxation properties.

In this article, we investigate the effect of $S \rightarrow S-1$ contributions to the magnetic anisotropy of high spin $\mathrm{Fe}$ (II) and $\mathrm{Co}$ (II) mononuclear complexes in various model geometries. The choice of these two ions is justified by the existence of a considerable number of single molecule magnets based on these metal centers [14,39-51]. Idealized structures of aquo complexes were built with the following coordination geometries: linear, trigonal planar, square planar, tetrahedral, trigonal bipyramidal, square pyramidal, and octahedral. Energies and wave functions for all models were obtained by CASSCF calculations, including the effect of SOC. Zero-field splitting parameters were decomposed in contributions from $S \rightarrow S$ and $S \rightarrow S-1$ excited states. For non-degenerate ground states, $S \rightarrow S$ and $S \rightarrow S-1$ contributions were of the same sign, and lower multiplicity states were responsible for $5-10 \%$ of the total magnetic anisotropy. This trend was observed for several coordination geometries and can be related to fundamental expressions for $D$. Then, the observed enhancement of magnetic anisotropy due to lower energy excited states should be encountered in real systems. In the case of degenerate ground states, the effect of $S-1$ excited states in the value of $D$ is minor $(<2 \%)$.

\section{Results and Discussion}

\section{1. $d$-Orbital Splitting}

As mentioned earlier, several studies have established connections between molecular structure and magnetic anisotropy in mononuclear complexes based on transition metal ions. Thus, it is reasonable to consider the $d$-orbital splitting as the starting point for the discussion. Figure 1 shows the energy splitting of all of the studied geometries based on ab initio ligand field theory (AILFT) [19] energies for the $d$-orbitals (see Materials and Methods section for further information). Figure 2 presents molecular geometries for model complexes.

It is important to stress that zero-field splitting parameters are obtained by a perturbative treatment of ground state interaction with excited levels due to the spin-orbit coupling operator. In this way, the ground state must be energetically isolated to allow for the treatment. For orbitally degenerate ground states, $D$ is undefined and ad hoc spin Hamiltonians must be developed for the case of interest.

In a strict sense, molecular complexes with high symmetry are scarce, as they require very simple ligands (monoatomic/linear) and the absence of distortions such as Jahn-Teller or crystal packing effects. In practice, complexes are described by reference shapes (e.g., square planar, octahedral) based on a similarity criterium for the metal center and donor atom positions. In most cases, many of the symmetry operations of the reference geometry are not present in the real compound. For the presented models, we always have a lower degree of symmetry due to the hydrogen atoms of the aquo ligands. 
A second set of geometries was constructed to analyze the effect of Jahn-Teller distortions. In some models, orbital degeneracy of the ground state is broken substantially by the geometric distortions, permitting the use of zero-field splitting parameters. Other examples develop a small energy splitting, which is insufficient for a perturbative treatment, and $D$ cannot be used as a valid parameter.

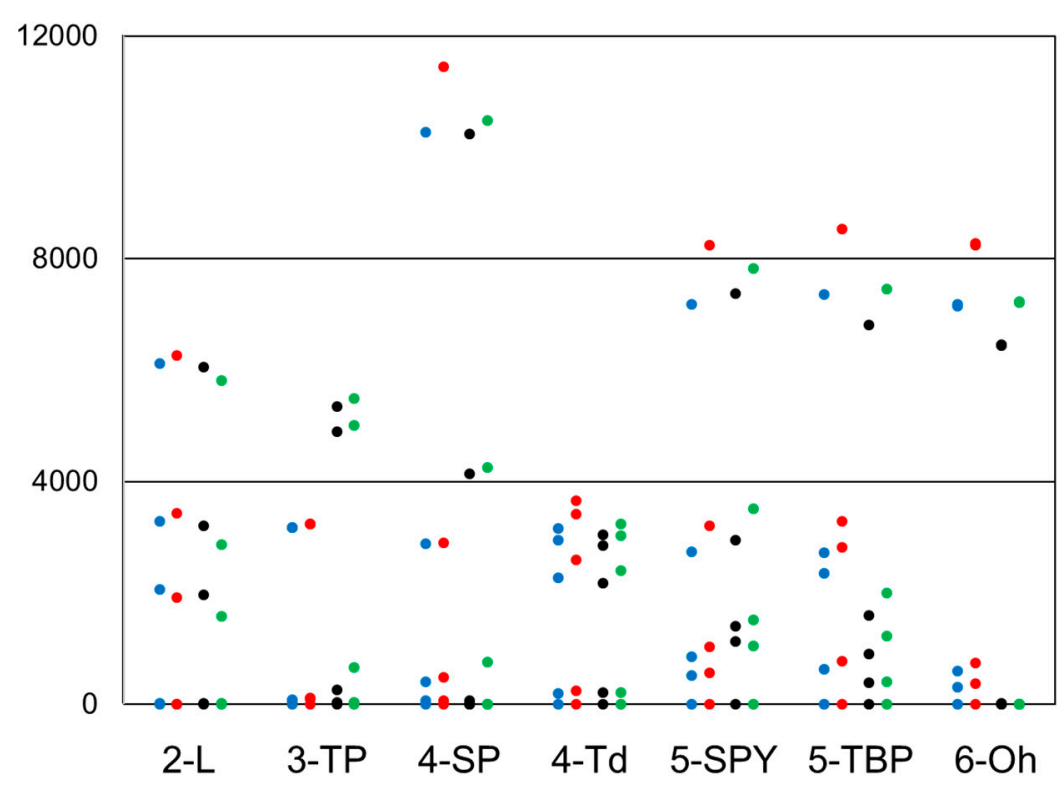

Figure 1. Ab initio ligand field theory (AILFT)-based $d$-orbital energy splitting $\left(\mathrm{cm}^{-1}\right)$ for complete active space self-consistent field (CASSCF) $(n, 5)$ and N-electron valence state perturbation theory (NEVPT2) for all Fe(II) (CASSCF: blue; NEVPT2: red) and Co(II) (CASSCF: black; NEVPT2: green) model structures. Calculations considered all highest multiplicity roots. Geometries are labeled using the code: 2-L (linear); 3-TP (trigonal planar); 4-SP (square planar); 4-Td (tetrahedral); 5-SPY (square pyramid); 5-TBP (trigonal bipyramid); 6-Oh (octahedral).
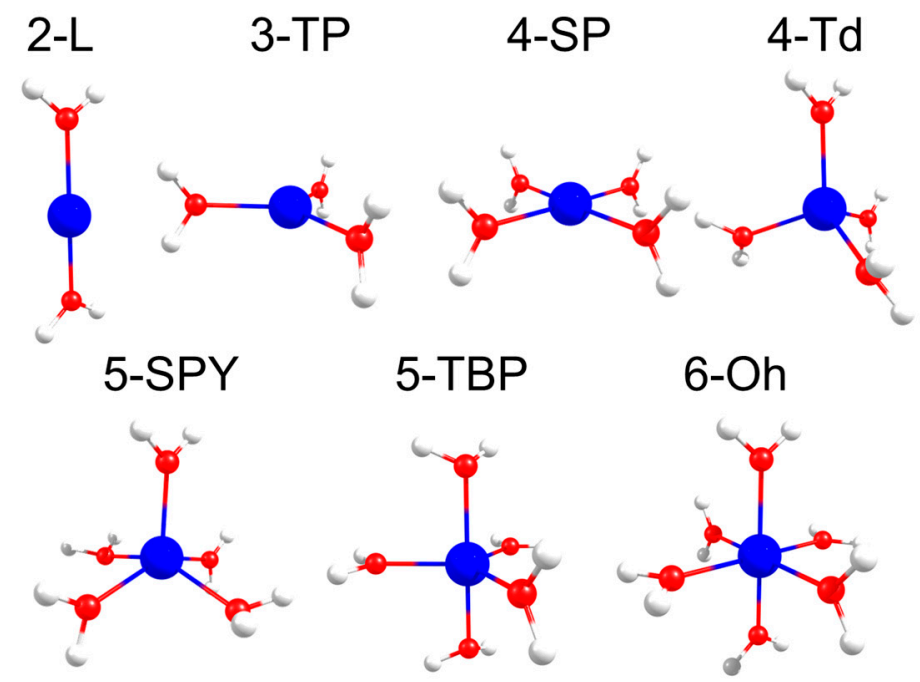

Figure 2. Molecular geometries for $\mathrm{Co}(\mathrm{II})$ model complexes. Structures are labeled using the code: 2-L (linear); 3-TP (trigonal planar); 4-SP (square planar); 4-Td (tetrahedral); 5-SPY (square pyramid); 5-TBP (trigonal bipyramid); 6-Oh (octahedral). Atom color code: Co (blue); O (red); H (white).

Models with a unique metal-oxygen distance are analyzed first. In the case of linear coordination, $d$-orbitals order in three blocks as expected. The most stable orbitals are $d_{x y}$ and $d_{x^{2}-y^{2}}$, which are separated by less than $20 \mathrm{~cm}^{-1}$ in all cases. The next block includes $d_{x z}$ and $d_{y z}$, which are significantly 
split by the lower symmetry due to hydrogen positions of the water ligands (ca. 1000-1500 $\mathrm{cm}^{-1}$ ). The highest energy is associated to the $d_{z^{2}}$ orbital. CASSCF energies indicate both Fe(II) and Co(II) models present degenerate ground states. Thus, their low energy spectrum cannot be described by the standard zero-field splitting parameters. In the case of $\mathrm{Fe}(\mathrm{II})$, the $d$-orbital splitting is consistent with the observed degeneracy, as the $d_{x y}^{2} d_{x^{2}-y^{2}}^{1} d_{x z}^{1} d_{y z}^{1} d_{z^{2}}^{1}$ and $d_{x y}^{1} d_{x^{2}-y^{2}}^{2} d_{x z}^{1} d_{y z}^{1} d_{z^{2}}^{1}$ configurations will be the most stable. In the case of $\mathrm{Co}(\mathrm{II})$, the interplay of interelectronic repulsion and ligand field splitting yields a doubly degenerate ground state, which wave functions present significant contributions from $d_{x y}^{2} d_{x^{2}-y^{2}}^{1} d_{x z}^{2} d_{y z}^{1} d_{z^{2}}^{1}, d_{x y}^{2} d_{x^{2}-y^{2}}^{1} d_{x z}^{1} d_{y z}^{2} d_{z^{2}}^{1}, d_{x y}^{1} d_{x^{2}-y^{2}}^{2} d_{x z}^{2} d_{y z}^{1} d_{z^{2}}^{1}$ and $d_{x y}^{1} d_{x^{2}-y^{2}}^{2} d_{x z}^{1} d_{y z}^{2} d_{z^{2}}^{1}$ configurations.

Trigonal planar geometry leads again to three orbital blocks: $\left(d_{z^{2}}\right),\left(d_{x z}, d_{y z}\right),\left(d_{x y}, d_{x^{2}-y^{2}}\right)$. The first three orbitals are close in energy. In the case of Fe(II), $d_{z^{2}}$ is the most stable where for Co(II) $d_{x z}$ and $d_{y z}$ are lower in energy. CASSCF energies show that the Fe(II) ground state is close to triply degenerate while the Co(II) first excited state appears at $1408.2 \mathrm{~cm}^{-1}$ (CASSCF). In this way, the Fe(II) low energy spectrum cannot de described by $D$ and $E$ while Co(II) is probably suitable for this kind of description. Formally, the ground state of $\mathrm{Co}(\mathrm{II})$ in a trigonal planar environment is orbitally degenerate, but the symmetry lowering due to hydrogen positions of aquo ligands breaks this symmetry.

Square planar geometry follows the text book ordering, with $\left(d_{x z}, d_{y z}\right)$ as the lowest to orbitals, followed by $d_{z^{2}}, d_{x y}$ and $d_{x^{2}-y^{2}}$. The existence of two $d$-orbitals with the lowest energy leads again to a degenerate ground state for $\mathrm{Fe}(\mathrm{II})$, given its $\mathrm{d}^{6}$ configuration, as the first excited state has an energy of $70 \mathrm{~cm}^{-1}$. For $\mathrm{Co}(\mathrm{II})$, the first excited state is at $1000-1200 \mathrm{~cm}^{-1}$. Thus, the ground state is non-degenerate, but there are low energy excited states. In this regime, zero-field splitting parameters are expected to be qualitatively able to describe magnetic anisotropy, but their values will be dependent of the method of choice (second-order perturbation theory or effective Hamiltonian) and largely sensitive to small changes in state energies (for instance, by N-electron valence state perturbation theory (NEVPT2) correction). Thus, we are in a borderline situation where $D$ values can be informative but must be analyzed with caution.

Tetrahedral coordination shows the characteristic $e, t_{2}$ splitting, with some splitting inside each block due to hydrogen positions. The splitting of the $t_{2}$ block is larger than the energy separation for the $e$ levels, due to the antibonding nature of the $t_{2}$ orbitals. As mentioned earlier, having a set of two $d$-orbitals as the most stable will lead to a degenerate ground state for high spin $d^{6}$ (Fe(II)), but not for $\mathrm{d}^{7}(\mathrm{Co}(\mathrm{II}))$. In this way, the first excited state for the iron model is at ca. $200 \mathrm{~cm}^{-1}$, while Co(II) presents an energy gap over $2000 \mathrm{~cm}^{-1}$, allowing the use of $D$ to describe its magnetic anisotropy.

Square pyramid coordination shows a slightly differing behavior, according to AILFT orbitals. In the case of $\mathrm{Fe}(\mathrm{II}), d_{x y}, d_{x z}$ and $d_{y z}$ appear close in energy and significantly mixed. For Co(II), the splitting between these orbitals is larger. In both cases, highest energy orbitals are $d_{z^{2}}$ and $d_{x^{2}-y^{2}}$. As three $d$-orbitals are close to be the lowest in energy, several configurations can be stabilized and several CASSCF states appear close to the ground state, although the ground state is still non-degenerate for both ions. First excitation energies are $526.2 \mathrm{~cm}^{-1}$ (Fe(II), CASSCF) and $664.5 \mathrm{~cm}^{-1}$ (Co(II), CASSCF). NEVPT2 results are qualitatively similar and are presented in Table S1. Under such small energy gap, the ability of $D$ values to represent the low energy spectrum of the system is limited, and other spin Hamiltonian parameters such as effective g-values of each Kramers' doublet can be especially useful for the description of the system.

Trigonal bipyramid spitting leads to three blocks of orbitals. The most stable orbitals are $\left(d_{x z}, d_{y z}\right)$, followed by $\left(d_{x y}, d_{x^{2}-y^{2}}\right)$ and finally $d_{z^{2}}$. Both $\left(d_{x z}, d_{y z}\right)$ and $\left(d_{x y}, d_{x^{2}-y^{2}}\right)$ are significantly split by symmetry lowering from hydrogen atoms (between $400-600 \mathrm{~cm}^{-1}$ ). Low energy excitations are in the same range as the observed for 5-SPY geometry. For instance, first excited states for CASSCF appear at $638.0 \mathrm{~cm}^{-1}$ for $\mathrm{Fe}(\mathrm{II})$ and $588.4 \mathrm{~cm}^{-1}$ for $\mathrm{Co}(\mathrm{II})$. Similar to the case of square pyramid coordination, $D$ will be in an intermediate range between the perturbative regime and a degenerate ground state.

Finally, octahedral geometry shows the typical $t_{2 g}, e_{g}$ splitting. In the case of Co(II), orbital blocks are essentially degenerate while Fe(II) shows a larger splitting. As expected when three $d$-orbitals 
are close to be the lowest in energy, both models will present low excitation energies consistent with a degenerate ground state. From CASSCF energies, Fe(II) has a state at $299.3 \mathrm{~cm}^{-1}$ and Co(II) at $4.2 \mathrm{~cm}^{-1}$. Therefore, $D$ will not be suitable to describe these systems.

\subsection{Effect of Jahn-Teller Distortion}

In the development of single molecule magnets, Jahn-Teller distortions are often an unwanted effect, since they lift orbital degeneracies which favor the development of unquenched orbital angular momentum in the ground state, associated with magnetic anisotropy. To obtain a set of models including Jahn-Teller distortion, we repeated all optimizations, allowing for the relaxation of metal-oxygen distances, but constraining $\mathrm{O}-\mathrm{M}-\mathrm{O}$ angles to remain representative of the reference geometry.

Orbital splitting was affected to a varying degree by the inclusion of Jahn-Teller distortion. Of course, linear systems remain unaffected, although a small energy difference is observed due to the change in metal-oxygen distances. In terms of CASSCF energies, it is clear how first excitation energies are raised due to Jahn-Teller distortion (See Table 1). Thus, zero-field splitting parameters for these geometries will be closer to the perturbational regime than undistorted models.

Table 1. First excitation energy $\left(\mathrm{cm}^{-1}\right)$ for CASSCF calculations, including all roots for all multiplicities stemming from for $\mathrm{d}^{6}\left(\mathrm{Fe}^{\mathrm{II}}\right)$ and $\mathrm{d}^{7}\left(\mathrm{CO}^{\mathrm{II}}\right)$ configurations. Geometries including and excluding Jahn-Teller distortion are labeled as "JT" and "noJT", respectively. Geometries with fixed (noJT) and relaxed (JT) metal-oxygen distances are presented as Supporting Information.

\begin{tabular}{ccccc}
\hline \multirow{2}{*}{ Geometry } & \multicolumn{2}{c}{ Fe(II) } & \multicolumn{2}{c}{ Co(II) } \\
\cline { 2 - 5 } & noJT & JT & noJT & JT \\
\hline 2-L & 8.9 & 26.3 & 7.3 & 3.8 \\
3-TP & 63.7 & 609 & 1408.2 & 2217.8 \\
4-SP & 68.8 & 565.1 & 1000.5 & 1195.9 \\
4-Td & 203.4 & 304.3 & 2268.5 & 3016.5 \\
5-SPY & 526.2 & 970.9 & 664.5 & 1155.2 \\
5-TBP & 638 & 913.8 & 588.4 & 2196.3 \\
6-Oh & 299.3 & 461.6 & 4.2 & 394.7 \\
\hline
\end{tabular}

\subsection{Adequacy of Zero-Field Splitting Parameters}

As discussed earlier, $D$ and $E$ parameters are adequate to describe magnetic anisotropy as long as the ground state is isolated enough in energy. When the ground state is degenerate or exhibits low energy excited states, $D$ is not a sensible parameter anymore and ad hoc spin Hamiltonians should be constructed for each case. Expectedly, calculated $D$ values under these circumstances are not robust, yielding unphysical values which are strongly sensitive to the method and source energies (CASSCF or NEVPT2). Table 2 presents $D$ values obtained for all model systems employing the second-order perturbation theory and effective Hamiltonian approaches. All roots of all multiplicities were included. It is clear that robust results are obtained only when the ground state is isolated enough to permit the perturbative regime defining the zero-field splitting parameters.

In the case of $\mathrm{Co}(\mathrm{II})$, zero-field splitting parameters are clearly unsuitable for linear and octahedral geometry, as evidenced by the unphysical values obtained by second-order perturbation theory (2PT) and the strongly dissimilar values provided by the effective Hamiltonian $\left(\mathrm{H}_{\mathrm{eff}}\right)$ approach. For the remaining geometries, $D_{\text {Heff }}$ values tend to be significantly smaller than $D_{2 \mathrm{PT}}$ results, although $E / D$ parameters are generally consistent between both approaches. This shows the existence of a regime where the ground state is non-degenerate but there are important low energy excited states. As expected, Jahn-Teller distorted geometries show smaller changes between $D_{2 \mathrm{PT}}$ and $D_{\text {Heff }}$.

For Fe(II), reasonable geometries to analyze $D$ are 5-SPY and 5-TBP. The sign change of $D$ observed for 5-SPY geometry may suggest that this coordination is not fulfilling the requirements for the 
calculation of $D$. However, the high rhombic component of the system dilutes the meaning of the sign of $D$ (considering CASSCF results, $E / D$ is 0.28 and 0.31 for $2 \mathrm{PT}$ and $\mathrm{H}_{\text {eff }}$ methods, respectively). In the case of Jahn-Teller distorted geometries, we find that most geometries (except linear) show similar values for $D_{2 \mathrm{PT}}$ and $D_{\text {Heff }}$.

Table 2. $D\left(\mathrm{~cm}^{-1}\right)$ and $E / D$ parameters calculated for all models using second-order perturbation theory $(2 \mathrm{PT})$ and effective Hamiltonian $\left(\mathrm{H}_{\text {eff }}\right)$ approaches. CASSCF calculation including all roots for every possible multiplicity stemming from $d$-orbital configurations.

\begin{tabular}{cccccccccc}
\hline \multicolumn{7}{c}{ Fe(II) } \\
\hline \multirow{6}{*}{ noJT } & 2-L & -4799.7 & -83.1 & 0.00 & 0.01 & -36459.3 & -239.8 & 0.00 & 0.00 \\
& 3-TP & 475.9 & 21.4 & 0.05 & 0.02 & 133.8 & 79.1 & 0.02 & 0.02 \\
& 4-SP & -438.4 & -37.7 & 0.03 & 0.26 & 160.2 & 87.0 & 0.00 & 0.00 \\
& 4-Td & -10.1 & -4.9 & 0.10 & 0.06 & -18.6 & -14.6 & 0.25 & 0.24 \\
& 5-SPY & -22.1 & 18.0 & 0.28 & 0.31 & -185.3 & -117.9 & 0.17 & 0.21 \\
& 5-TBP & -11.1 & -10.1 & 0.24 & 0.15 & 83.7 & 62.7 & 0.02 & 0.15 \\
& 6-Oh & -33.0 & 25.8 & 0.23 & 0.24 & 15175.6 & 175.0 & 0.03 & 0.02 \\
\hline JT & 2-L & -1602.8 & -82.5 & 0.00 & 0.00 & -64329.6 & -236.0 & 0.00 & 0.00 \\
& 3-TP & -49.0 & -33.4 & 0.09 & 0.00 & 86.6 & 64.5 & 0.00 & 0.00 \\
& 4-SP & -54.6 & -38.0 & 0.02 & 0.04 & 134.5 & 82.9 & 0.00 & 0.00 \\
& 4-Td & -8.1 & -5.0 & 0.02 & 0.09 & -15.7 & -13.4 & 0.24 & 0.24 \\
& 5-SPY & -7.5 & -6.1 & 0.30 & 0.27 & -82.2 & -66.6 & 0.26 & 0.26 \\
& 5-TBP & 7.8 & -6.7 & 0.29 & 0.32 & 50.7 & 42.0 & 0.02 & 0.02 \\
& 6-Oh & 22.1 & 21.9 & 0.28 & 0.16 & 143.5 & 116.4 & 0.01 & 0.00 \\
\hline
\end{tabular}

As the objective of this article is to study the contributions of the same and different spin excitations to magnetic anisotropy, we want to keep as many cases as possible to draw the most general conclusions. In this way, only strongly differing cases in terms of $D_{2 \mathrm{PT}}$ and $D_{\text {Heff }}$ will be discarded due to the inapplicability of zero-field splitting parameters in orbitally degenerate ground states. To give a clear selection criterium, we chose $D_{\text {Heff }} / D_{2 \mathrm{PT}}>0.5$ as the condition to accept a geometry. Of course, if the ratio between both $D$ values is close to 0.5 , predictions about energy splitting and magnetic anisotropy will be significantly dependent on the method chosen to calculate the zero-field splitting parameters and conclusions should be drawn carefully.

\subsection{Inclusion of Lower Multiplicity Roots}

The effect of lower multiplicity roots in magnetic anisotropy will be analyzed for the geometries with non-degenerate ground states. In the next section, cases with degenerate ground states will be analyzed separately. Table 3 presents the values for $D$ and $E / D$, considering only the highest multiplicity roots or all roots in the CASSCF calculation. We only show results for the second-order perturbation theory method. Effective Hamiltonian values are presented in Table S2.

In general, the inclusion of all roots resulted in a rise in the magnitude of $D$. For CASSCF calculations, the majority of cases clustered around $5 \%$ to $10 \%$. There are some exceptions, such as Fe(II)-5-TBP (noJT), where $D_{\text {All }}$ is slightly lower than $D_{\text {HS }}$. Three models presented a rise exceeding $10 \%$. In most cases, NEVPT2 calculations also show a rise in $D$ upon the inclusion of roots from all multiplicities, with a broader range for the $D_{\mathrm{All}} / D_{\mathrm{HS}}$ ratio. This change is not related to differences in the orbital optimization from the CASSCF calculation, as $D$ and $E / D$ values are not changing when the configuration interaction is performed with the orbitals optimized for all roots or only the highest multiplicity roots (see Table S3). 
Table 3. $D\left(\mathrm{~cm}^{-1}\right)$ and $E / D$ values for selected models of Fe(II) and Co(II). Parameters correspond to CASSCF $(n, 5)$ calculations including highest multiplicity roots (HS index) or all roots for every possible multiplicity stemming from $d$-orbital configurations (All index).

\begin{tabular}{ccccccccccc}
\hline & & & \multicolumn{3}{c}{ CASSCF } & \multicolumn{3}{c}{ NEVPT2 } \\
\hline \multirow{2}{*}{ Fe(II) } & noJT & 5-SPY & -22.1 & 0.28 & -21.0 & 0.29 & -19.9 & 0.25 & -19.0 & 0.27 \\
& noJT & 5-TBP & -11.1 & 0.24 & -11.5 & 0.14 & -8.8 & 0.27 & -9.4 & 0.15 \\
& JT & 3-TP & -49.0 & 0.09 & -48.1 & 0.09 & -44.6 & 0.07 & -32.5 & 0.01 \\
& JT & 4-SP & -54.6 & 0.02 & -53.1 & 0.02 & -54.2 & 0.00 & -36.5 & 0.02 \\
& JT & 4-Td & -8.1 & 0.02 & -6.2 & 0.15 & -7.7 & 0.04 & -3.8 & 0.06 \\
& JT & 5-SPY & -7.5 & 0.30 & -6.2 & 0.29 & 7.0 & 0.32 & -5.6 & 0.22 \\
& JT & 5-TBP & 7.8 & 0.29 & -7.3 & 0.25 & 6.7 & 0.26 & -7.1 & 0.19 \\
& JT & 6-Oh & 22.1 & 0.28 & 21.6 & 0.23 & 18.1 & 0.32 & 21.8 & 0.13 \\
\hline \multirow{2}{*}{ Co(II) } & noJT & 3-TP & 133.8 & 0.02 & 125.9 & 0.02 & 108.3 & 0.03 & 102.9 & 0.03 \\
& noJT & 4-SP & 160.2 & 0.00 & 151.4 & 0.00 & 135.5 & 0.00 & 127.3 & 0.00 \\
& noJT & 4-Td & -18.6 & 0.25 & -17.4 & 0.24 & -14.8 & 0.27 & -13.9 & 0.27 \\
& noJT & 5-SPY & -185.3 & 0.17 & -177.8 & 0.15 & -147.7 & 0.17 & -145.9 & 0.15 \\
& noJT & 5-TBP & 83.7 & 0.02 & 76.4 & 0.02 & 69.4 & 0.01 & 63.4 & 0.01 \\
& JT & 3-TP & 86.6 & 0.00 & 78.4 & 0.00 & 73.3 & 0.00 & 58.2 & 0.00 \\
& JT & 4-SP & 134.5 & 0.00 & 124.7 & 0.00 & 109.3 & 0.00 & 77.1 & 0.00 \\
& JT & 4-Td & -15.7 & 0.24 & -14.4 & 0.23 & -13.1 & 0.25 & -12.3 & 0.23 \\
& JT & 5-SPY & -82.2 & 0.26 & -78.9 & 0.23 & -62.0 & 0.28 & -64.4 & 0.24 \\
& JT & 5-TBP & 50.7 & 0.02 & 43.5 & 0.02 & 42.3 & 0.02 & 36.2 & 0.02 \\
\hline
\end{tabular}

Table 4 shows the decomposition of the zero-field splitting parameter $\left(D_{\text {All }}\right)$ in contributions from the highest multiplicity $\left(D_{\text {High }}\right)$ and lower multiplicity roots $\left(D_{\text {Low }}\right)$. These values are compared to the calculation including only the highest multiplicity roots (named " $D_{\mathrm{HS}}$ " in Table 4). In general, $D_{\mathrm{HS}}$ tends to be closer to $D_{\mathrm{High}}$ than $D_{\mathrm{All}}$, indicating that the contribution from same spin excitation remains similar, independent of the roots included in the CASSCF. In this way, the differences between $D_{\mathrm{HS}}$ and $D_{\mathrm{All}}$ can be mainly attributed to excitations between states of different multiplicities. It is interesting to see that $D_{\text {High }}$ and $D_{\text {Low }}$ are of the same sign in all studied cases, and their relative weight is in many cases in the range between $5-10 \%$. There are some cases where the contributions of $D_{\text {Low }}$ represent a larger fraction of $D_{\text {All }}$, such as in Co(II)-5-TBP (JT), Fe(II)-4-Td, 5-SPY, and 5-TBP (JT).

The contribution of lower multiplicity roots to zero-field splitting parameters was always of the same sign of the total value. Moreover, it always led to a rise in magnetic anisotropy. As mentioned, the contribution of different-spin excitations to $D$ was of a similar order in most cases, around $5-10 \%$ of the total value. To understand this behavior, it is instructive to recall the expression for the zero-field splitting parameter for excitations between states of the same spin $\left(D_{K L}^{S O C-(0)}\right)$ and when the spin is lowered by one unit $\left(D_{K L}^{S O C-(-1)}\right)[52,53]$.

$$
\begin{gathered}
D_{K L}^{S O C-(0)}=-\frac{1}{S^{2}} \sum_{I \prime(S I=S)} \Delta_{J \prime}^{-1}\left\langle\Psi_{0}^{S S}\left|\sum_{i} \hat{z}_{K}(i) \hat{s}_{0}(i)\right| \Psi_{J \prime}^{S S}\right\rangle\left\langle\Psi_{J \prime}^{S S}\left|\sum_{i} \hat{z}_{L}(i) \hat{s}_{0}(i)\right| \Psi_{0}^{S S}\right\rangle \\
D_{K L}^{S O C-(-1)}=-\frac{1}{S(2 S-1)} \sum_{I \prime(S I=S-1)} \Delta_{J \prime}^{-1}\left\langle\Psi_{0}^{S S}\left|\sum_{i} \hat{z}_{K}(i) \hat{s}_{+1}(i)\right| \Psi_{J \prime}^{S-1 S-1}\right\rangle\left\langle\Psi_{J \prime}^{S-1 S-1}\left|\sum_{i} \hat{z}_{L}(i) \hat{s}_{-1}(i)\right| \Psi_{0}^{S S}\right\rangle
\end{gathered}
$$

where $S$ is the spin, $K, L$ are cartesian components $(x, y$ or $z) . \Delta_{J^{\prime}}^{-1}$ is the reciprocal of the energy difference between the ground state $\left(\Psi_{0}^{S S}\right)$ and the excited state of interest $\left(\Psi_{J I}^{S S}\right) . \hat{z}_{L}(i)$ is part of the spin-orbit coupling operator $\hat{z}_{L}(i) \hat{s}_{L}(i)$ for electron $i$, with respect to the $L$ cartesian component. $\hat{s}_{0, \pm 1}(i)$ are the components of the spin vector operator.

To simplify the problem, we will assume that states can be expressed in terms of single determinants. Thus, $\hat{z}_{L}(i)$ is decomposed in the product of an effective SOC constant $\left(\xi_{e f f}\right)$ and 
the corresponding angular momentum operator $\left(\hat{l}_{K, b a}(i)\right)$, connecting the initial $\left(d_{b}\right)$ and final $\left(d_{a}\right)$ orbitals describing the excited electron. The matrix elements appearing in Equations (1) and (2) will be expressed as:

$$
\xi_{e f f}\left\langle d_{a, i}\left|\sum_{i} \hat{l}_{K, b a}(i) \hat{s}_{m}(i)\right| d_{b, i}\right\rangle
$$

where $m=-1,0,1$. Considering $\hat{l}_{K, b a}(i)$ is imaginary and $\hat{l}_{K, b a}(i)=-\hat{l}_{K, a b}(i)$, it is easy to see that $D_{K K}^{S O C-(0)}$ will be negative and $D_{K K}^{S O C-(-1)}$ will be positive. As $D_{\text {High }}$ and $D_{\text {Low }}$ tended to show the same sign, the largest component in absolute value for $D_{\text {High }}$ must be the smallest for $D_{\text {Low }}$. This is indeed corroborated in CASSCF calculations (see Table S4). This relation will only hold for $x x, y y$, and $z z$ components $D$. Mixed components for $D_{K L}^{S O C-(-1)}$ can be positive or negative, potentially leading to a different behavior. At least in our models, diagonal elements of the $D$ tensor tended to dominate over non-diagonal values, yielding overall positive contributions to $D^{S O C-(-1)}$.

Table 4. $D\left(\mathrm{~cm}^{-1}\right)$ for selected geometries under different choices of the multiplicity of included roots. $D$ values in the "All" section correspond to CASSCF calculations including all roots. $D_{\text {All }}$ is the total value, which is decomposed in contributions from highest multiplicity $\left(D_{\text {High }}\right)$ and lower multiplicity roots $\left(D_{\text {Low }}\right)$. The calculation considering only higher multiplicity roots is in the section HS. Results for the second-order perturbation theory method for the calculation of zero-field splitting parameters are presented.

\begin{tabular}{ccccccccccc}
\hline \multicolumn{1}{c}{} & \multicolumn{9}{c}{ CASSCF } & \multicolumn{4}{c}{ NEVPT2 } \\
\hline \multirow{2}{*}{ Fe(II) } & noJT & 5-SPY & -22.1 & -20.3 & -1.8 & -21.0 & -19.9 & -18.0 & -1.9 & -19.0 \\
& noJT & 5-TBP & -11.1 & -11.1 & 0.0 & -11.5 & -8.8 & -8.6 & -0.2 & -9.4 \\
& JT & 3-TP & -49.0 & -46.9 & -2.1 & -48.1 & -44.6 & -42.4 & -2.2 & -32.5 \\
& JT & 4-SP & -54.6 & -52.1 & -2.6 & -53.1 & -54.2 & -51.4 & -2.8 & -36.5 \\
& JT & 4-Td & -8.1 & -6.2 & -1.9 & -6.2 & -7.7 & -5.8 & -1.9 & -3.8 \\
& JT & 5-SPY & -7.5 & -5.8 & -1.8 & -6.2 & 7.0 & 5.3 & 1.7 & -5.6 \\
& JT & 5-TBP & 7.8 & 6.3 & 1.5 & -7.3 & 6.7 & 5.1 & 1.6 & -7.1 \\
& JT & 6-Oh & 22.1 & 21.2 & 1.0 & 21.6 & 18.1 & 17.1 & 1.0 & 21.8 \\
\hline \multirow{2}{*}{ Co(II) } & noJT & 3-TP & 133.8 & 125.2 & 8.6 & 125.9 & 108.3 & 99.5 & 8.8 & 102.9 \\
& noJT & 4-SP & 160.2 & 149.9 & 10.3 & 151.4 & 135.5 & 123.8 & 11.7 & 127.3 \\
& noJT & 4-Td & -18.6 & -17.5 & -1.2 & -17.4 & -14.8 & -13.6 & -1.2 & -13.9 \\
& noJT & 5-SPY & -185.3 & -180.0 & -5.4 & -177.8 & -147.7 & -142.1 & -5.7 & -145.9 \\
& noJT & 5-TBP & 83.7 & 76.1 & 7.6 & 76.4 & 69.4 & 61.5 & 7.9 & 63.4 \\
& JT & 3-TP & 86.6 & 77.8 & 8.8 & 78.4 & 73.3 & 64.3 & 9.0 & 58.2 \\
& JT & 4-SP & 134.5 & 123.1 & 11.4 & 124.7 & 109.3 & 95.6 & 13.7 & 77.1 \\
& JT & 4-Td & -15.7 & -14.4 & -1.3 & -14.4 & -13.1 & -11.7 & -1.3 & -12.3 \\
& JT & 5-SPY & -82.2 & -79.2 & -3.1 & -78.9 & -62.0 & -58.6 & -3.4 & -64.4 \\
& JT & 5-TBP & 50.7 & 43.2 & 7.5 & 43.5 & 42.3 & 34.5 & 7.8 & 36.2 \\
\hline
\end{tabular}

The $\mathrm{d}^{6}$ system is taken as example. For a negative value of $D, D_{z z}<1 / 2\left(D_{x x}+D_{y y}\right)$. Then, the most important contribution to $D$ is associated with orbitals connected by the $z$ component of the angular momentum operator. For $D_{K K}^{S O C-(0)}$, the excitation will correspond to the promotion of the only beta electron from the doubly occupied orbital in the ground state to a singly occupied orbital. Initial and final orbitals will be called ' 1 ' and ' 2 ', and the matrix element will be $l_{z, 12}$. In the case of $D_{K K}^{S O C-(-1)}$, the electron must promote to an excited $d$-orbital and spin flip. Thus, the final orbital must be initially empty in order to accommodate the electron. For each $S=S^{\prime}$ excitation (e.g., $21111 \rightarrow 12111$ ), there will be three lower multiplicity determinants contributing to $D_{K L}^{S O C-(-1)}$ (i.e., 22110, 22101, 22011). The relevant matrix elements will be $l_{K, 23}, l_{K, 24}$ and $l_{K, 25}$. If " 1 " and " 2 " are already connected by the $z$ component, it is likely that " 2 " is connected to " 3 ", " 4 ", and " 5 " by the $x$ or $y$ components. In this way, $D_{z z}^{S O C-(-1)}$ will be smaller than $D_{x x}^{S O C-(-1)}$ and $D_{y y}^{S O C-(-1)}$, in accordance with calculations. 


\subsection{Degenerate Ground States}

As zero-field splitting parameters are not directly applicable for these systems, the influence of lower multiplicity roots will be analyzed in terms of state energies after the inclusion of spin-orbit coupling. Table 5 presents the lowest energy levels for the geometries not considered in the previous section. As one of the key properties in single molecule magnet research is the excitation energy to overcome the top of the energy barrier produced by the splitting of the $\mathrm{M}_{\mathrm{s}}$ components of the ground state, we consider this energy as the parameter to evaluate the effect of the inclusion of excitations to lower multiplicity states. In the case of Fe(II), it corresponds to the fifth state, and for Co(II) it is the second Kramers' doublet. In the majority of cases, energy barrier energies are modified less than $2 \%$ upon the inclusion of lower multiplicity roots. This is significantly smaller than the observed changes in $D$ parameters and is expected since the dominant contribution for magnetic anisotropy in degenerate systems comes from the interaction within the ground state levels. Thus, contributions from higher excited states will be of minor importance. The exception from this behavior is Fe(II)-4-Td, which exhibits a very low energy barrier (c.a. $20 \mathrm{~cm}^{-1}$ ), and small changes in energy give large percentage modifications.

Table 5. State energies $\left(\mathrm{cm}^{-1}\right)$ after the inclusion of spin-orbit coupling effects (QDPT) for selected geometries (CASSCF calculations). Due to Kramers' degeneracy, all states are doubly degenerate for $\mathrm{Co}(\mathrm{II})$, so we only show each energy once.

\begin{tabular}{|c|c|c|c|c|c|c|}
\hline & & All & HS & & All & HS \\
\hline \multirow[t]{15}{*}{$\mathrm{Fe}(\mathrm{II})$} & 2-L (noJT) & 0.0 & 0.0 & 4-Td (noJT) & 0.0 & 0.0 \\
\hline & & 0.0 & 0.0 & & 1.2 & 0.7 \\
\hline & & 193.8 & 190.9 & & 14.8 & 10.2 \\
\hline & & 193.8 & 190.9 & & 15.7 & 14.0 \\
\hline & & 388.0 & 385.1 & & 20.5 & 16.0 \\
\hline & 3-TP (noJT) & 0.0 & 0.0 & 6-Oh (noJT) & 0.0 & 0.0 \\
\hline & & 19.0 & 18.7 & & 5.9 & 6.3 \\
\hline & & 21.0 & 21.2 & & 39.8 & 37.7 \\
\hline & & 84.8 & 84.7 & & 101.9 & 101.2 \\
\hline & & 84.8 & 84.8 & & 110.6 & 108.9 \\
\hline & 4-SP (noJT) & 0.0 & 0.0 & 2-L (JT) & 0.0 & 0.0 \\
\hline & & 0.6 & 0.6 & & 0.0 & 0.0 \\
\hline & & 104.5 & 102.3 & & 195.8 & 192.9 \\
\hline & & 113.7 & 111.5 & & 196.0 & 193.0 \\
\hline & & 195.5 & 194.1 & & 381.5 & 379.3 \\
\hline \multirow[t]{8}{*}{$\mathrm{Co}(\mathrm{II})$} & 2-L (noJT) & 0.0 & 0.0 & 2-L (JT) & 0.0 & 0.0 \\
\hline & & 479.6 & 470.9 & & 472.0 & 464.2 \\
\hline & & 960.9 & 954.7 & & 941.7 & 937.5 \\
\hline & & 1444.5 & 1451.2 & & 1423.3 & 1433.3 \\
\hline & 6-Oh (noJT) & 0.0 & 0.0 & 6-Oh (JT) & 0.0 & 0.0 \\
\hline & & 350.3 & 350.1 & & 232.9 & 234.4 \\
\hline & & 353.0 & 352.8 & & 512.5 & 512.2 \\
\hline & & 933.8 & 947.7 & & 960.8 & 969.7 \\
\hline
\end{tabular}

\section{Materials and Methods}

\section{Computational Details}

All calculations were performed using the ORCA 4.0.1.2 software package [54]. Model structures were constructed using ideal geometries for the $\mathrm{MO}_{n}$ fragment, while hydrogen positions were fully optimized for all cases. Metal-oxygen distances were set to the average of unconstrained optimizations for high spin hexa-aquo complexes of iron(II) (2.151 $\AA$ ) and cobalt(II) (2.113 $\AA$ ). To discuss the effect of Jahn-Teller bond elongation/compression, a second set of molecules was optimized without 
restrictions on metal-oxygen bond distances. The BP86 density functional $[55,56]$ and the Def2-TZVP basis set $[57,58]$ were employed in all geometry optimizations. We considered a CASSCF [59] active space involving the five $3 \mathrm{~d}$ orbitals, following common practice in single-molecule magnet research. N-electron valence state perturbation theory (NEVPT2) $[60,61]$ energy correction was also included. Converged CASSCF wave functions served as a basis to build the state interaction matrix of the spin-orbit coupling (SOC) operator (quasi degenerate perturbation theory (QDPT) approach). Zero-field splitting parameters ( $D$ and $E$ ) obtained by second-order perturbation theory and effective Hamiltonian approaches were compared, and $d$-orbital splitting was analyzed in terms of ab initio ligand field theory (AILFT) [19]. In all cases, CASSCF and NEVPT2 ground states corresponded to the highest multiplicity computed (quintet for Fe(II) and quartet for Co(II)).

\section{Conclusions}

The effect of lower multiplicity roots in zero-field splitting parameters was evaluated for model structures of $d^{6}(\mathrm{Fe}(\mathrm{II}))$ and $\mathrm{d}^{7}(\mathrm{Co}(\mathrm{II}))$ complexes. When the ground state was energetically isolated, lower multiplicity states increased the total $D$ value by ca. $5-10 \%$ in most cases. This trend should be observed in general and is related to the sign of the contributions of same spin and different spin excitations. In the case of degenerate ground states, the effect of high energy roots was less important, with contributions under $2 \%$. This behavior is expected since spin-orbit coupling between levels of the ground state will greatly dominate the magnetic anisotropy. Conclusions drawn for model systems are expected to hold in real molecular systems given the correspondence of the observed trend with the basic equations describing zero-field splitting parameters.

Supplementary Materials: Tables S1-S4 and Cartesian coordinates of all calculated models are available online at ww.mdpi/2304-6740/6/1/24/s1. Table S1: First non-relativistic excitation energies for all models, Table S2: $D$ values obtained by effective Hamiltonian method, Table S3: $D$ values with differently optimized orbitals, Table S4: D-tensors for $S \rightarrow S$ and $S \rightarrow S-1$ contributions

Acknowledgments: The authors thank FONDECYT Regular 1170524 project for financial support. Powered@NLHPC: This research was partially supported by the supercomputing infrastructure of the NLHPC (ECM-02) (Leftraru and UsachHPC servers).

Author Contributions: Leonel Llanos performed the electronic structure calculations. Daniel Aravena conceived the project and wrote the paper. Both authors analyzed the data.

Conflicts of Interest: The authors declare no conflict of interest.

\section{References}

1. Ishikawa, N.; Sugita, M.; Ishikawa, T.; Koshihara, S.Y.; Kaizu, Y. Lanthanide double-decker complexes functioning as magnets at the single-molecular level. J. Am. Chem. Soc. 2003, 125, 8694-8695. [CrossRef] [PubMed]

2. Habib, F.; Luca, O.R.; Vieru, V.; Shiddiq, M.; Korobkov, I.; Gorelsky, S.I.; Takase, M.K.; Chibotaru, L.F.; Hill, S.; Crabtree, R.H.; et al. Influence of the ligand field on slow magnetization relaxation versus spin crossover in mononuclear cobalt complexes. Angew. Chem. Int. Ed. 2013, 52, 11290-11293. [CrossRef] [PubMed]

3. Chen, Y.; Liu, J.-L.; Ungur, L.; Liu, J.; Li, Q.; Wang, L.; Ni, Z.; Chibotaru, L.F.; Chen, X.-M.; Tong, M. Symmetry-Supported Magnetic Blocking at $20 \mathrm{~K}$ in Pentagonal Bipyramidal Dy(III) Single-Ion Magnets. J. Am. Chem. Soc. 2016, 138, 2829-2837. [CrossRef] [PubMed]

4. $\quad$ Ding, Y.-S.; Chilton, N.F.; Winpenny, R.E.P.; Zheng, Y.-Z. On Approaching the Limit of Molecular Magnetic Anisotropy: A Near-Perfect Pentagonal Bipyramidal Dysprosium(III) Single-Molecule Magnet. Angew. Chem. Int. Ed. 2016, 55, 16071-16074. [CrossRef] [PubMed]

5. Goodwin, C.A.P.; Ortu, F.; Reta, D.; Chilton, N.F.; Mills, D.P. Molecular magnetic hysteresis at 60 kelvin in dysprosocenium. Nature 2017, 548, 439-442. [CrossRef] [PubMed]

6. Díaz-Torres, R.; Menelaou, M.; Roubeau, O.; Sorrenti, A.; Brandariz-de-Pedro, G.; Sañudo, E.C.; Teat, S.J.; Fraxedas, J.; Ruiz, E.; Aliaga-Alcalde, N. Multiscale study of mononuclear Co(II) SMMs based on curcuminoid ligands. Chem. Sci. 2016, 7, 2793-2803. [CrossRef] 
7. Aravena, D.; Ruiz, E. Shedding Light on the Single-Molecule Magnet Behavior of Mononuclear Dy(III) Complexes. Inorg. Chem. 2013, 52, 13770-13778. [CrossRef] [PubMed]

8. Atanasov, M.; Zadrozny, J.M.; Long, J.R.; Neese, F. A theoretical analysis of chemical bonding, vibronic coupling, and magnetic anisotropy in linear iron(II) complexes with single-molecule magnet behavior. Chem. Sci. 2013, 4, 139-156. [CrossRef]

9. Rechkemmer, Y.; Breitgoff, F.D.; Van Der Meer, M.; Atanasov, M.; Hakl, M.; Orlita, M.; Neugebauer, P.; Neese, F.; Sarkar, B.; Slageren, J. Van A four-coordinate cobalt(II) single-ion magnet with coercivity and a very high energy barrier. Nat. Commun. 2016, 7, 1-8. [CrossRef] [PubMed]

10. Cahier, B.; Maurice, R.; Bolvin, H.; Mallah, T.; Guihéry, N. Tools for Predicting the Nature and Magnitude of Magnetic Anisotropy in Transition Metal Complexes: Application to Co(II) Complexes. Magnetochemistry 2016, 2, 31. [CrossRef]

11. Campbell, V.E.; Bolvin, H.; Rivière, E.; Guillot, R.; Wernsdorfer, W.; Mallah, T. Structural and electronic dependence of the single-molecule-magnet behavior of dysprosium(III) complexes. Inorg. Chem. 2014, 53, 2598-2605. [CrossRef] [PubMed]

12. El-Khatib, F.; Cahier, B.; López-Jordà, M.; Guillot, R.; Rivière, E.; Hafez, H.; Saad, Z.; Girerd, J.-J.; Guihéry, N.; Mallah, T. Design of a Binuclear Ni(II) Complex with Large Ising-type Anisotropy and Weak Anti-Ferromagnetic Coupling. Inorg. Chem. 2017, 56, 10655-10663. [CrossRef] [PubMed]

13. Cahier, B.; Perfetti, M.; Zakhia, G.; Naoufal, D.; El-Khatib, F.; Guillot, R.; Rivière, E.; Sessoli, R.; Barra, A.L.; Guihéry, N.; et al. Magnetic anisotropy in pentacoordinate Ni(II) and Co(II) complexes: Unraveling electronic and geometrical contributions. Chem. A Eur. J. 2017, 23, 3648-3657. [CrossRef] [PubMed]

14. Vaidya, S.; Upadhyay, A.; Singh, S.K.; Gupta, T.; Tewary, S.; Langley, S.K.; Walsh, J.P.S.; Murray, K.S.; Rajaraman, G.; Shanmugam, M. A synthetic strategy for switching the single ion anisotropy in tetrahedral Co(II) complexes. Chem. Commun. 2015, 51, 3739-3742. [CrossRef] [PubMed]

15. Gupta, S.K.; Rajeshkumar, T.; Rajaraman, G.; Murugavel, R. An air-stable Dy(III) single-ion magnet with high anisotropy barrier and blocking temperature. Chem. Sci. 2016, 7, 5181-5191. [CrossRef]

16. Belio Castro, A.; Jung, J.; Golhen, S.; Le Guennic, B.; Ouahab, L.; Cador, O.; Pointillart, F. Slow Magnetic Relaxation in Unprecedented Mono-Dimensional Coordination Polymer of Ytterbium Involving Tetrathiafulvalene-Dicarboxylate Linker. Magnetochemistry 2016, 2, 26. [CrossRef]

17. Zhang, P.; Jung, J.; Zhang, L.; Tang, J.; Le Guennic, B. Elucidating the Magnetic Anisotropy and Relaxation Dynamics of Low-Coordinate Lanthanide Compounds. Inorg. Chem. 2016, 55, 1905-1911. [CrossRef] [PubMed]

18. Ribbing, C.; Pierloot, K.; Ceulemans, A. Ab Initio Calculations of the Trigonal and Zero-Field Splittings in Trischelated Diketonato Complexes of Trivalent Chromium. Inorg. Chem. 1998, 37, 5227-5232. [CrossRef]

19. Atanasov, M.; Ganyushin, D.; Sivalingam, K.; Neese, F.; Atanasov, M.; Ganyushin, D.; Sivalingam, K.; Neese, F. A Modern First-Principles View on Ligand Field Theory Through the Eyes of Correlated Multireference Wavefunctions. In Molecular Electronic Structures of Transition Metal Complexes II; Mingos, D.M.P., Day, P., Dahl, J.P., Eds.; Structure and Bonding; Springer: Berlin/Heidelberg, Germany, 2012; ISBN 978-3-642-27377-3.

20. Chibotaru, L.F. Ab Initio Methodology for Pseudospin Hamiltonians of Anisotropic Magnetic Complexes. In Advances in Chemical Physics; Rice, S.A., Dinner, A.R., Eds.; John Wiley \& Sons, Inc: Hoboken, NJ, USA, 2013; Volume 153, ISBN 978-1-118-57176-7.

21. Chibotaru, L.F.; Ungur, L. Ab initio calculation of anisotropic magnetic properties of complexes. I. Unique definition of pseudospin Hamiltonians and their derivation. J. Chem. Phys. 2012, 137, 64112. [CrossRef] [PubMed]

22. Aravena, D.; Atanasov, M.; Neese, F. Periodic Trends in Lanthanide Compounds through the Eyes of Multireference ab Initio Theory. Inorg. Chem. 2016, 55, 4457-4469. [CrossRef] [PubMed]

23. Schweinfurth, D.; Sommer, M.G.; Atanasov, M.; Demeshko, S.; Hohloch, S.; Meyer, F.; Neese, F.; Sarkar, B. The Ligand Field of the Azido Ligand: Insights into Bonding Parameters and Magnetic Anisotropy in a Co(II)-Azido Complex. J. Am. Chem. Soc. 2015, 137, 1993-2005. [CrossRef] [PubMed]

24. García-Fuente, A.; Cimpoesu, F.; Ramanantoanina, H.; Herden, B.; Daul, C.; Suta, M.; Wickleder, C.; Urland, W. A ligand field theory-based methodology for the characterization of the $\mathrm{Eu}^{2+}[\mathrm{Xe}] 4 \mathrm{f}^{6} 5 \mathrm{~d}^{1}$ excited states in solid state compounds. Chem. Phys. Lett. 2015, 622, 120-123. [CrossRef]

25. Gatteschi, D.; Sessoli, R. Quantum Tunneling of Magnetization and Related Phenomena in Molecular Materials. Angew. Chem. Int. Ed. 2003, 42, 268-297. [CrossRef] [PubMed] 
26. Gómez-Coca, S.; Urtizberea, A.; Cremades, E.; Alonso, P.J.; Camón, A.; Ruiz, E.; Luis, F. Origin of slow magnetic relaxation in Kramers ions with non-uniaxial anisotropy. Nat. Commun. 2014, 5, 4300. [CrossRef] [PubMed]

27. Pedersen, K.S.; Dreiser, J.; Weihe, H.; Sibille, R.; Johannesen, H.V.; Sørensen, M.A.; Nielsen, B.E.; Sigrist, M.; Mutka, H.; Rols, S.; et al. Design of Single-Molecule Magnets: Insufficiency of the Anisotropy Barrier as the Sole Criterion. Inorg. Chem. 2015, 54, 7600-7606. [CrossRef] [PubMed]

28. Lunghi, A.; Totti, F.; Sanvito, S.; Sessoli, R. Intra-molecular origin of the spin-phonon coupling in slow-relaxing molecular magnets. Chem. Sci. 2017, 8, 6051-6059. [CrossRef] [PubMed]

29. Woods, T.J.; Ballesteros-Rivas, M.F.; Gómez-Coca, S.; Ruiz, E.; Dunbar, K.R. Relaxation Dynamics of Identical Trigonal Bipyramidal Cobalt Molecules with Different Local Symmetries and Packing Arrangements: Magnetostructural Correlations and ab inito Calculations. J. Am. Chem. Soc. 2016, 138, 16407-16416. [CrossRef] [PubMed]

30. Atanasov, M.; Comba, P.; Helmle, S.; Müller, D.; Neese, F. Zero-Field Splitting in a Series of Structurally Related Mononuclear Ni(II)-Bispidine Complexes. Inorg. Chem. 2012, 51, 12324-12335. [CrossRef] [PubMed]

31. Duboc, C.; Collomb, M.-N.; Neese, F. Understanding the Zero-Field Splitting of Mononuclear Manganese(II) Complexes from Combined EPR Spectroscopy and Quantum Chemistry. Appl. Magn. Reson. 2010, 37, 229-245. [CrossRef]

32. Maurice, R.; de Graaf, C.; Guihéry, N. Magnetostructural relations from a combined ab initio and ligand field analysis for the nonintuitive zero-field splitting in Mn(III) complexes. J. Chem. Phys. 2010, 133, 84307. [CrossRef] [PubMed]

33. Perić, M.; García-Fuente, A.; Zlatar, M.; Daul, C.; Stepanović, S.; García-Fernández, P.; Gruden-Pavlović, M. Magnetic anisotropy in "scorpionate" first-row transition-metal complexes: A theoretical investigation. Chem. A Eur. J. 2015, 21, 3716-3726. [CrossRef] [PubMed]

34. Gomez-Coca, S.; Cremades, E.; Aliaga-Alcalde, N.; Ruiz, E. Mononuclear Single-Molecule Magnets: Tailoring the Magnetic Anisotropy of First-Row Transition-Metal Complexes. J. Am. Chem. Soc. 2013, 135, 7010-7018. [CrossRef] [PubMed]

35. Stavretis, S.E.; Atanasov, M.; Podlesnyak, A.A.; Hunter, S.C.; Neese, F.; Xue, Z.-L. Magnetic Transitions in Iron Porphyrin Halides by Inelastic Neutron Scattering and Ab Initio Studies of Zero-Field Splittings. Inorg. Chem. 2015, 54, 9790-9801. [CrossRef] [PubMed]

36. Aravena, D.; Venegas-Yazigi, D.; Ruiz, E. Single-Molecule Magnet Properties of Transition-Metal Ions Encapsulated in Lacunary Polyoxometalates: A Theoretical Study. Inorg. Chem. 2016, 55, 6405-6413. [CrossRef] [PubMed]

37. Neese, F.; Solomon, E.I. Calculation of Zero-Field Splittings, $g$-Values, and the Relativistic Nephelauxetic Effect in Transition Metal Complexes. Application to High-Spin Ferric Complexes. Inorg. Chem. 1998, 37, 6568-6582. [CrossRef] [PubMed]

38. Sato, R.; Suzuki, K.; Minato, T.; Shinoe, M.; Yamaguchi, K.; Mizuno, N. Field-induced slow magnetic relaxation of octahedrally coordinated mononuclear Fe(III)-, Co(II)-, and Mn(III)-containing polyoxometalates. Chem. Commun. 2015, 51, 4081-4084. [CrossRef] [PubMed]

39. Gómez-Coca, S.; Aravena, D.; Morales, R.; Ruiz, E. Large magnetic anisotropy in mononuclear metal complexes. Coord. Chem. Rev. 2015, 289-290, 379-392. [CrossRef]

40. Freedman, D.E.; Harman, W.H.; Harris, T.D.; Long, G.J.; Chang, C.J.; Long, J.R. Slow magnetic relaxation in a high-spin iron(II) complex. J. Am. Chem. Soc. 2010, 132, 1224-1225. [CrossRef] [PubMed]

41. Weismann, D.; Sun, Y.; Lan, Y.; Wolmershäuser, G.; Powell, A.K.; Sitzmann, H. High-Spin Cyclopentadienyl Complexes: A Single-Molecule Magnet Based on the Aryl-Iron(II) Cyclopentadienyl Type. Chem. A Eur. J. 2011, 17, 4700-4704. [CrossRef] [PubMed]

42. Zadrozny, J.M.; Liu, J.; Piro, N.A.; Chang, C.J.; Hill, S.; Long, J.R. Slow magnetic relaxation in a pseudotetrahedral cobalt(II) complex with easy-plane anisotropy. Chem. Commun. 2012, 48, 3927-3929. [CrossRef] [PubMed]

43. Mathonière, C.; Lin, H.-J.; Siretanu, D.; Clérac, R.; Smith, J.M. Photoinduced Single-Molecule Magnet Properties in a Four-Coordinate Iron(II) Spin Crossover Complex. J. Am. Chem. Soc. 2013, 135, 19083-19086. [CrossRef] [PubMed] 
44. Eichhöfer, A.; Lan, Y.; Mereacre, V.; Bodenstein, T.; Weigend, F. Slow magnetic relaxation in trigonal-planar mononuclear Fe(II) and $\mathrm{Co}(\mathrm{II})$ bis(trimethylsilyl)amido complexes-A comparative study. Inorg. Chem. 2014, 53, 1962-1974. [CrossRef] [PubMed]

45. Novikov, V.V.; Pavlov, A.A.; Nelyubina, Y.V.; Boulon, M.-E.; Varzatskii, O.A.; Voloshin, Y.Z.; Winpenny, R.E.P. A Trigonal Prismatic Mononuclear Cobalt(II) Complex Showing Single-Molecule Magnet Behavior. J. Am. Chem. Soc. 2015, 137, 9792-9795. [CrossRef] [PubMed]

46. Craig, G.A.; Murrie, M. 3D Single-Ion Magnets. Chem. Soc. Rev. 2015, 44, 2135-2147. [CrossRef] [PubMed]

47. Habib, F.; Korobkov, I.; Murugesu, M. Exposing the intermolecular nature of the second relaxation pathway in a mononuclear cobalt(II) single-molecule magnet with positive anisotropy. Dalt. Trans. 2015, 44, 6368-6373. [CrossRef] [PubMed]

48. Mondal, A.K.; Khatua, S.; Tomar, K.; Konar, S. Field-Induced Single-Ion-Magnetic Behavior of Octahedral CoII in a Two-Dimensional Coordination Polymer. Eur. J. Inorg. Chem. 2016, 2016, 3525-3552. [CrossRef]

49. Frost, J.M.; Harriman, K.L.M.; Murugesu, M. The rise of 3-d single-ion magnets in molecular magnetism: Towards materials from molecules? Chem. Sci. 2016, 7, 2470-2491. [CrossRef] [PubMed]

50. Zhang, Y.-Z.; Gómez-Coca, S.; Brown, A.J.; Saber, M.R.; Zhang, X.; Dunbar, K.R. Trigonal antiprismatic Co(II) single molecule magnets with large uniaxial anisotropies: Importance of Raman and tunneling mechanisms. Chem. Sci. 2016, 7, 6519-6527. [CrossRef] [PubMed]

51. Zadrozny, J.M.; Greer, S.M.; Hill, S.; Freedman, D.E. A flexible iron(II) complex in which zero-field splitting is resistant to structural variation. Chem. Sci. 2016, 7, 416-423. [CrossRef]

52. Atanasov, M.; Aravena, D.; Suturina, E.; Bill, E.; Maganas, D.; Neese, F. First principles approach to the electronic structure, magnetic anisotropy and spin relaxation in mononuclear $3 \mathrm{~d}$-transition metal single molecule magnets. Coord. Chem. Rev. 2015, 289, 177-214. [CrossRef]

53. Ganyushin, D.; Neese, F. First-principles calculations of zero-field splitting parameters. J. Chem. Phys. 2006, 125, 24103. [CrossRef] [PubMed]

54. Neese, F. Software update: The ORCA program system, version 4.0. Wiley Interdiscip. Rev. Comput. Mol. Sci. 2017, 8, e1327. [CrossRef]

55. Becke, A.D. Density-functional exchange-energy approximation with correct asymptotic behavior. Phys. Rev. A 1988, 38, 3098-3100. [CrossRef]

56. Perdew, J.P. Density-functional approximation for the correlation energy of the inhomogeneous electron gas. Phys. Rev. B 1986, 33, 8822-8824. [CrossRef]

57. Weigend, F.; Ahlrichs, R. Balanced basis sets of split valence, triple zeta valence and quadruple zeta valence quality for H to Rn: Design and assessment of accuracy. Phys. Chem. Chem. Phys. 2005, 7, 3297-3305. [CrossRef] [PubMed]

58. Weigend, F. Accurate Coulomb-fitting basis sets for H to Rn. Phys. Chem. Chem. Phys. 2006, 8, 1057-1065. [CrossRef] [PubMed]

59. Malmqvist, P.-Å.; Roos, B.O. The CASSCF state interaction method. Chem. Phys. Lett. 1989, 155, $189-194$. [CrossRef]

60. Angeli, C.; Cimiraglia, R.; Evangelisti, S.; Leininger, T.; Malrieu, J.-P. Introduction of n -electron valence states for multireference perturbation theory. J. Chem. Phys. 2001, 114, 10252-10264. [CrossRef]

61. Angeli, C.; Cimiraglia, R.; Malrieu, J.-P. N-electron valence state perturbation theory: A fast implementation of the strongly contracted variant. Chem. Phys. Lett. 2001, 350, 297-305. [CrossRef]

(C) 2018 by the authors. Licensee MDPI, Basel, Switzerland. This article is an open access article distributed under the terms and conditions of the Creative Commons Attribution (CC BY) license (http://creativecommons.org/licenses/by/4.0/). 